\title{
El Cuervo de Edgar Allan Poe: ingenio y poesía ${ }^{1}$
}

\author{
José SILES ARTÉS \\ jsartes@gmail.com
}

Recibido: septiembre 2010

Aceptado: marzo 2011

\section{RESUMEN}

Este artículo parafrasea cada una de las 18 estrofas de El Cuervo de Edgar Allan Poe, para mostrar el proceso narrativo del poema. Al mismo tiempo, se señalan los diferentes recursos empleados por el poeta al objeto de crear una atmósfera de misterio y terror, haciendo de contrafondo el reino de la muerte.

Palabras clave: Nunca jamás, muerte, terror, pesadilla, siniestra, umbral, desventurado, fantasmal, despiadado, desenlace.

\section{Edgar Allan Poe's “The Raven": wit and poetry}

\section{ABSTRACT}

This article paraphrases each of the 18 stanzas of Edgar Allan Poe's The Raven, in order to display its narrative process. At the same time, mention is made of the different specific ways used by the poet to create an atmosphere of mystery and terror, with the world beyond the grave acting as.a background.

Keywords: Nevermore, death, terror, nightmare, sinister, threshold, unfortunate, ghostly, merciless, outcome.

\section{Le Corbeau d'Edgar Allan Poe: génie et poésie}

\section{RÉSUMÉ}

Cet article paraphrase les 18 strophes de Le corbeau d'Edgar Allan Poe pour montrer le processus narratif du poème. En même temps, on fait remarquer les divers procédés employés par le poète afin de créer une atmosphère de mystère et de terreur, avec le royaume de la mort comme toile de fond.

Mots-clés : jamais plus, mort, terreur, cauchemar, sinistre, seuil, malheureux, fantomatique, sans pitié, dénouement.

SUMARIO: 1. Introducción. 2. Proceso narrativo y recursos. Referencias bibliográficas.

${ }^{1}$ Conferencia dada en el Ateneo de Madrid, el 16 de noviembre de 2009, con motivo del Segundo Centenario del nacimiento de Poe. 


\section{INTRODUCCIÓN}

Edgar Allan Poe(1809-1849) es un clásico indiscutible, y prueba de ello es la edición que de sus obras se hace sin cesar. No hay más que buscar su nombre en el catálogo de cualquier biblioteca. Otros ocupan un lugar tan relevante como el suyo en la historia de la literatura, pero pocos conservan la actualidad indeclinable de Poe. Nada tiene de extraordinario este desequilibrio, que se corresponde justamente con el legado de Poe. Sus cuentos constituyen la mayor parte de su producción literaria, y además tuvieron un éxito y marcaron unas rutas que han hecho época. Sus ideas sobre la poesía han recibido y siguen recibiendo atención, aunque siempre dentro del reducido círculo de los teóricos de la literatura.

No estoy apuntando a que su poesía haya sido injustamente dada de lado o no apreciada; más bien creo que su reedición y estudio es el que le corresponde, pequeño por relación al potente corpus de su prosa.

Poe en realidad no escribió más que unos cincuenta poemas, pertenecientes a cuatro libros de poesía, cu yos títulos conocen los especialistas, pero no normalmente los simples lectores. El simple lector, el lector de a pie, recuerda al poeta por un puñado de poemas, cuya, relevancia $\mathrm{h}$ a traspasado todas las fronteras. Entre ellos, además de "El Cuervo", están "To Helen"(1831 ), The City in the Sea"(1831), "Lenore"(1831), "The Raven"(1845), "Ulalume”(1847), “Annabel Lee”(1849) y “The Bells"(1849).

"El Cuervo" apareció por primera vez el 29 de enero de 1845, en un periódico de Nueva York, el Evening Mirror , causando un impacto inmediato y dando a su autor una gran notoriedad. En las generaciones posteriores el éxito y el conocimiento de este poema se ha mantenido, situándose en el mismo rango por lo menos que los afamados y novedosos relatos de su autor. En 1856 la obra de Poe causó sensación en Francia, al aparecer sus cuentos, bajo el título de Histoires Extraordinaires, traducidos y lúcidamente prologados por Baudelaire, quien en 1858 daba a la luz una segunda recopilación denominada, Nouvelles Histoires Extraordinaires. La admiración y dedicación de Baudelaire a la obra de Poe fue decisiva para el conocimiento de éste en Europa y, concretamente, para el desarrollo de la escuela simbolista.

No podía Baudelaire dejar de lado El Cuervo, el más afamado poema de su ídolo americano, pero curiosamente lo tradujo en prosa. Como Mallarmé, años después. En España, como era de suponer, la obra de Poe se empieza a conocer a través del francés, y es Pedro Antonio de Alarcón quien, inspirándose en Baudelaire, se adelanta con un artículo publicado en La Época en septiembre de 1858. Es la señal de salida de un conocimiento y admiración que tendrá su paralelo en Hispanoamérica. Y precisamente será un venezolano, Juan Antonio Pérez Bonalde, quien en 1887 publica su afortunada traducción rimada de El Cuervo, que todavía se lee con placer.

Más adelante, en 1893, Poe es definitivamente consagrado en el mundo de habla española, cuando Rubén Darío le dedica un artículo en La Nación de Buenos Aires, como señala Eugene Englekirk en Edgar Allan Poe in Hispanic Literature (Instituto de Las Españas en los Estados Unidos(New York, 1934), libro fundamental, como también lo es, con un alcance más concreto, la obra de Santiago Rodríguez GuerreroStrachan, Presencia de Edgar Allan Poe en la literatura español del siglo XIX 
(Valladolid: Secretariado de Publicaciones e Intercambio Científico, Universidad de Valladolid, 1999). El Cuervo tuvo un éxito inmediato y clamoroso, haciéndole sobrepasar al escritor la fama de que ya gozaba por sus famosos cuentos. El poema se convirtió en una pieza popular que se aprendía y se recitaba de memoria, hasta el punto de que por la calle, los niños seguían a Poe y le recitaban estrofas de su poema, respondiendo él festivamente con su famoso estribillo de nevermore.

Es sabido que una pieza de tan gran aceptación apenas le dio beneficio a su autor, en línea con el escaso rédito que obtuvo con sus demás obras. Poe vivió en perpetua penuria económica. Cuando un escritor, un artista, acierta en ganar la atención y el interés general con una obra en particular, lo normal es que produzca otra u otras del mismo estilo para aprovechar el éxito. Pero Poe, con El Cuervo, no se decantó por esta vía, sino que capitalizó su éxito de estas dos maneras: Una, juntando el poema con otros varios y publicándolos bajo el título de The Raven and Other Poems (1845). Dos, publicando un minucioso comentario sobre el mismo, The Philosophy of Composition, que apareció en el Graham's Magazine en 1846.

De la lectura del susodicho escrito se desprende que la palabra filosofía no está usada en un sentido universal, genérico, clásico, significando "amor al saber", especulación, ejercicio del pensamiento, sino en el más concreto de "arte", "manera o reglas para hacer una cosa", en este caso la composición de su poema, El Cuervo.

Y de él hace resaltar un a serie de motivos y procedimientos que serían los más idóneos para forjar el poema ideal. Nos dice que su poema es el resultado de un plan preconcebido, inspirado en las más genuinas esencias de la poesía: belleza, aflicción, muerte y amor. Por otro lado, señala el empleo del ritmo trocaico a lo largo de la composición, así como el recurso a la aliteración y, exhaustivamente, al estribillo.

Evidentemente el comentario del poeta explicita la tramoya del hechizo, el revés del tapiz, pero en última instancia surge la pregunta de si El Cuervo fue gestado de manera tan racional, o si el comentario, el análisis, es una reflexión, a posteriori. En cualquier caso, a cada lector le cabe hacer su propio comentario. El mío figura a continuación, deteniéndose en cada una de las dieciocho estrofas que lo componen.

\section{PROCESO NARRATIVO Y RECURSOS}

El poema se abre con un estudioso que, estando medio dormido ("nearly napping") frente a sus libros, o ye un ruido como de alguien tocando levemente a su puerta, pero no estando del todo seguro, le quita importancia al hecho con la frase, "Only this and nothing more"("Sólo eso y nada más"), dicha sin duda para tranquilizarse.

Once upon a midnight dreary, while I pondered, weak and weary.

Over many a quaint and curious volume of forgotten lore,

While I nodded, nearly napping, suddenly there came a tapping,

As of someone gently rapping, rapping at my chamber door. 
" 'Tis some visitor, I muttered, "tapping at my chamber doorOnly this and nothing more."

En la segunda estrofa nuestra atención es desviada hacia el escenario del suceso, hacia el fuego de la chimenea y las espectrales formas que pinta sobre el suelo, dándonos a conocer seguidamente la profunda aflicción del poeta por la pérdida, la muerte, de Lenore, doncella de gran hermosura. No usa ahí Poe la palabra "muerte", pero que Lenore no está ya entre los vivos, que es un ser del más allá, de ultratumba, que vive entre los ángeles, queda muy claro ("... the radiant maiden whom the angels name Leno re"). Noticia que el verso final remach a: "Nameless here for evermore".

Ah, distinctly I remember it was in the bleak December, And each separate dying ember wrought its ghost upon the floor. Eagerly I wished the morrow; vainly I had sought to borrow From my books surcease of sorrow-sorrow for the lost Lenore, For the rare and radiant maiden whom the angels name LenoreNameless here for evermore.

En la tercera estrofa la velada intranquilidad, el implícito desasosiego de la primera, dan paso literalmente a una confesión de verdadero terror ("fantastic terrors never felt before"), tan fuerte y tan justificado, que su insistencia en la presencia de un visitante nos parece tan ilusoria como el poeta quiere hacernos ver. La causa tiene que ser otra, y además muy siniestra.

An the silken, sad uncertain rustling of each purple curtain Thrilled me-filled me with fantastic terrors never felt before; So that now, to still the beating on my heart, I stood repeating, "T is some visitor entreating entrance at my chamber doorSome late visitor entreating entrance at my chamber doorThis it is and nothing more."

En el cuarto movimiento, saca fuerzas de flaqueza el narrador ("Presently my soul grew stronger, hesitating then no longer") y se dirige, habla a un supuesto visitante; le pide perdón por su vacilación (“... truly your forgiveness I implore”) y abre la puerta, encontrándose con el negro silencio de la noche por toda presencia("Darkness there, and nothing more"). Hasta ahora todo es incierto, impreciso, intangible, misterioso. 
Presently my soul grew stronger: hesitating then no longer, "Sir", said I, "or Madam, truly your forgiveness I implore; But the fact is I was napping, and so gently you came rapping, And so faintly you came tapping, tapping at my chamber door, That I scarce was sure I heard you."-here I opened wide the doorDarkness there and nothing more.

Hasta que, en la quinta estrofa, se oye susurrar la palabra Lenore, que nuestro personaje repite y que es además duplicada por el eco. Es un punto en que ha empezado a oírse, por así decirlo, la banda sonora del poema. Al mismo tiempo, la tuerca del miedo ha dado una vuelta decisiva, Esa voz que desde las sombras de la noche se o ye es, en el contexto del suceso, signo de que algo paranormal o extraterreno está su cediendo, pues Lenore está muerta, habita en regiones de ultratumba. Estamos rozando la frontera del más allá, la estamos pisando, sensación acentuada por el irónico broche de la estrofa, "Merely this and nothing more"("Sólo esto y nada más").

Deep into that darkness, peering, long I stood there, wondering, fearing,

Doubting, dreaming dreams no mortal ever dared to dream before;

But the silence was unbroken, and the stillness gave no token, And the only word there spoken was the whispered word "Lenore!" This I whispered, and an echo murmured back the word "Lenore!" Merely this and nothing more.

En la estrofa siguiente, el narrador se ve enfrentado bruscamente a una ineludible materialización de su pesadilla. Si los espeluznantes signos descritos pertenecían todavía al terreno de las sospechas, de las figuraciones, ahora los supuestos toques se hacen más fuertes ("I heard a tapping somewhat louder than before"), innegables, contra la ventana de su estudio-al inicio habían sonado contra la puerta. Pero ¿quién es el autor? ¿El viento? "El viento y nada más", vaticina quiere creer el cuitado. Pero la revelación es inminente, estamos en un punto crítico, cabiendo varias soluciones más o menos plausibles dentro del contexto fabulado por el poeta; como la aparición de un hombre o mujer más o menos misterioso, pero de carne y hueso, o quizá de un espíritu, como el de la dulce Lenore.

Back into the chamber turning, all my soul within me burning, Soon again I heard a tapping, somewhat louder than before. 
"Surely," said I, "surely that is something at my window lattice;

Let me see, then, what thereat is, and this mystery explore,-

let my heart be still a moment and this mystery explore-

" $\mathrm{T}$ is the wind and nothing more."

Catapultado a la séptima tirada para salir de dudas, el lector no se va encontrar con ninguna de aquellas dos respuestas, sino que imprevisible y bruscamente presenciará la entrada en escena de un ave negra, un ave siniestra por excelencia, un gran cuervo. En la sabiduría del habla castellana su mala fama está popularmente condensada en el dicho, Cría cuervos y te sacarán los ojos. El intruso, de imponente apariencia, se instala sin vacilar en lugar altanero, concluyendo la estrofa con el por sexta vez repetido, y nada más, que implícitamente está alertando sobre la ocurrencia de "mucho más".

7

Open here I flung the shutter, when, with many a flirt and flutter, In there stepped a stately Raven of the saintly days of yore.

Not the least obeisance made he, not a minute stopped or stayed he,

But with mien of lord or lady perched above my chamber door-

Perched upon a bust of Pallas just above my chamber door-

Perched and sat, and nothing more.

En el clima sobrenatural en que estamos inmersos, ese cuervo tiene todos los visos de ser algo más que un pájaro. ¿No será la encarnación de un espíritu, de un ser del otro mundo? ¿No será una infernal figura del reino de Plutón? "Tell me what thy lordly name is on the Night's Plutonian shore", le demanda el protagonista. "Nevermore" responde el cuervo, y su voz es como un eco del más allá.

8

Then, this ebony bird beguiling my sad fancy into smiling By the grave and stern decorum of the countenance it wore, "Though thy crest be shorn and shaven, thou," I said, "art sure no craven,

Ghastly, grim, and ancient Raven, wandering from the Nightly shore:

Tell me what thy lordly name is in the Night's Plutonian shore!"

Quoth the Raven, "Nevermore!"

Aunque acaba de franquear el umbral de la realidad, palpando una ominosa y ultraterrena oscuridad, el protagonista habla sin embargo a su visitante en tono jocoso, descreído, desafiante, no queriendo aceptar su fantasmagoría; con un desdén que 
trasluce una inútil defensa. Nada tan humano como esconder la cabeza bajo el ala en trances críticos.

Much I marvelled this ungainly fowl to hear discourse so plainly, Though its answer little meaning, little relevancy, bore;

For we cannot help agreeing that no human being

Ever yet was blessed with seeing bird above his chamber door-

Bird or beast upon the sculptured bust above his chamber door-

With such name as "Nevermore."

En la inercia de su vana esperanza, el desventurado susurra que al llegar la mañana el intruso habrá desaparecido, acabando así su pesadilla, pero justamente ahí se oye de nuevo la voz del cuervo sentenciando, "nevermore", haciendo subir de punto nuestra perplejidad.

But the Raven, sitting lonely on the placid bust, spoke only That one word, as if his soul in that one word he did outpour, Nothing farther then he uttered, not a feather then he fluttered; Till I scarcely more than muttered, "Other friends have flown before;

On the morrow he will leave me, as my Hopes have flown before; Then the bird said, "Nevermore."

La muy coherente respuesta del cuervo en la estrofa 11 le sobrecoge, pero prefiere creer que la ominosa sentencia, el implacable "nevermore", no es más que una muletilla que algún amo le enseñó. No interviene el cuervo en ese episodio, tampoco lo hace en el 12, pero indeclinable en el mantenimiento del clima ultraterreno, el poeta recurre, si no al habla del intruso, sí a su diabólica presencia cu ando le describe como un "grim, ungainly, ghastly, gaunt and ominous bird of yore".

Startled at the stillness broken by reply so aptly spoken, "Doubtless, said I, "what it utters is its only stock and store, Caught from some unhappy master whom unmerciful Disaster Followed fast and followed faster till his songs one burden bore, Till the dirges of his Hope that melancholy burden bore Of 'Never-nevermore. " 


\section{2}

But the Raven still beguiling my sad fancy into smiling,

Straight I wheeled a cushioned seat in front of the bird and bust and door;

Then, upon the velvet sinking, I betook myself to linking

Fancy unto fancy, thinking what this ominous bird of yore,

What this grim, ungainly, ghastly gaunt, and ominous bird of yore, Meant in croaking "Nevermore."

Qué quiere decir el cuervo con su recurrente estribillo, se pregunta el infortunado, antes de evocar a su amada, la difunta Lenore, quien en este aposento ya no podrá reposar, "nunca jamás", cual sádicamente sentencia el cuervo. Y detrás de esa tortura, la insoslayable crueldad de la muerte, la ineluctable caída en el abismo de la nada, del "nada más". En la décimo tercera estrofa el ave permanece muda, fantasmal, posada en un prealudido busto de Pallas, atormentando con su mirada al desventurado: sus "fiery eyes now burned into my bosom's core". El cuervo aterroriza de tres maneras: con su habla, su presencia y sus ojos.

\section{3}

This I sat engaged in guessing, but no syllable expressing

To the fowl whose fiery eyes now burned into my bosom's core;

This and more I sat divining, with my head at ease reclining

On the cushion's velvet lining that the lamp-light gloated o'er,

But whose velvet violet lining, with the lamp-light gloating o'er,

She shall press, ah, nevermore!

El recuerdo de la amada alcanza tan alto grado de morbosa obsesión, que el amante ansía encontrar liberación: “jolvida a la difunta Lenora!”, se exhorta a sí mismo. "Nunca jamás", le responde inmediatamente su verdugo, dándole otro golpe despiadado que viene a decir: "no sólo has perdido a tu amada para siempre, sino que además nunca cesará tu tormento. Es particularmente pérfido aquí el cuervo, contrastando con la venturosa visión que el protagonista tiene de unos ángeles, cuyos pasos siente tintinear sobre el suelo: un oasis celestial en el poema.

\section{4}

Then, methought, the air grew denser, perfumed from an unseen censer

Swung by seraphim whose footfalls tinkled on the tufted floor.

"Wretch!" I cried, "thy God hath lent thee, by these angels he hath sent thee,

Respite-respite and nepenthe from thy memories of Lenore! 
Quaff, oh quaff this kind nepenthe, and forget this lost Lenore!" Quoth the Raven, "Nevermore."

¿Es un profeta el cuervo, es un ave? Cualquiera que sea su verdadera naturaleza, ¿puede decirle al desesperado amante, si verdaderamente hay una cura para su dolor, o en palabras del profeta Jeremías(8:22): “is there balm in Gilead?”, ¿hay bálsamo en Galaad?"

"Prophet" said I, "thing of evil! prophet still, if bird or devil! Whether Tempter sent, or whether tempest tossed thee here ashore, Desolate yet all undaunted, on this dessert land enchantedOn this home by Horror haunted-tell me truly I implore!, Is there- $i s$ there balm in Gilead?-tell me-tell me, I implore!" Quoth the Raven, "Never more."

"Prophet" y "thing of evil", repite, y tan sobrenatural vidente lo cree que hasta le pregunta si podrá abrazar a Lenore en el reino de los ángeles, en el remoto "Aidenn"(El Edén). Pero su respuesta, como fácilmente adivinamos, no va a ser otra que el negativo estribillo. No hay quizá en todo el poema un "nunca jamás" más desolador y sádico que éste. Atrás quedó la intriga sobre la identidad del visitante. Quedó zanjado que se trata d e un siniestro y demoníaco cuervo parlante.

\section{6}

"Prophet!" said I, "thing of evil! Prophet still, if bird or devil! By that Heaven that bends above us-by that God we both adoreTell this soul with sorrow laden if, within the distant Aidenn, It shall clasp a sainted maiden whom the angels name Lenore.l" Clasp a rare and radiant maiden whom the angels name Lenore." Quoth the Raven, "Nevermore."

En las tres estrofas precedentes es Lenore quien se convierte en el hilo maestro de la trama, pero claramente en la dieciséis queda agotado este recurso. ¿Qué intriga va a surgir a continuación? Lógicamente ninguna, no queda espacio, no quedan más que dos estrofas para la conclusión del poema. ¿Cómo lo va a instrumentar el poeta? Será bruscamente, por las bravas, como se ve en la penúltima tirada, donde el desventurado exige al cuervo que se vaya, que abandone su habitación. “... take thy form from off my do or!" Pero la respuesta está cantada, está cantada desde el inicio de la composición. No se va a ir, "nunca jamás". 


\section{7}

"Be that word our sign of parting, bird or fiend!" I shrieked, up starting;

"Get thee back into the tempest and the Night's Plutoniam shore! Leave no black plume as a token of that lie thy soul hath spoken!

Leave my loneliness unbroken!-quit the bust above my door!

Take thy beak from out my heart, and take thy form from off my door!"

Quoth the Raven, "Nevermore."

El clima de ultratumba y sobrenatural va por tanto a perpetuarse, así como el tormento del protagonista, que cierra el poema con imágenes aceradamente terroríficas, aludiendo por última vez al cuervo, mientras la luz de la lámpara proyecta su ominosa sombra sobre el suelo, sombra de la que nunca jamás se podrá liberar.

18

And the Raven, never flitting, still is sitting, still is sitting On the pallid bust of Pallas just above my chamber door; And his eyes have all the seeming of a demon's that is dreaming, And the lamp-light o'er him streaming throws his shadow on the floor;

And my soul from out that shadow that lies floating on the floor Shall be lifted-nevermore!

En realidad, desde la entrada del ave en la habitación no ha ocurrido nada, y nada sucede al final. Se podría decir que esta es una historia sin desenlace si la juzgáramos con una óptica narrativa, aunque desde el punto de vista poético puede concluirse que su desenlace consiste en que no hay desenlace, situándonos la última estrofa en el mirador de un abismo, el helado e inescrutable abismo de la muerte, donde no hay antes ni ahora, ni principio ni fin.

\section{REFERENCIAS BIBLIOGRÁFICAS}

ADAMS, John F. "Classical Raven Lore and Poe's Raven" in Poe Studies. Vol. V, no. 2, December 1972. Disponible online

FORSYTHE, Robert. "Poe's 'Nevermore': A Note", as collected in American Literature 7. January, 1936.

HOFFMAN, Daniel. Baton Rouge. ed. Poe Poe Poe Poe Poe Poe Poe. Louisiana State University Press. ISBN 08-071-2321-8. 
KRUTCH, Joseph Wood. Edgar Allan Poe: A Study in Genius. New York: Alfred A. Knopf, 1926.

POE, Edgar Allan. Edgar Allan Poe: Complete Tales \& Poems. Edison, NJ: Castle Books, 2002. ISBN 0-7858-1453-1 